J. Mawhin (Univ. Catholique de Louvain, Belgium)

\title{
RESONANCE AND NONLINEARITY: A SURVEY
}

\section{РЕЗОНАНС І НЕЛІНІЙНІСТЬ: ОГЛЯД}

This paper surveys recent results about nonresonant and resonant periodically forced nonlinear oscillators. This includes the existence of periodic, unbounded or bounded solutions for bounded nonlinear perturbations of linear and of piecewise linear oscillators, as well as of some classes of planar Hamiltonian systems.

Наведено огляд останніх результатів щодо нерезонансних і резонансних періодично збуджуваних нелінійних осциляторів: існування періодичних необмежених або обмежених розв' язківдля обмежених нелінійних збурень лінійних та кусково-лінійних осциляторів, а також деяких класів плоских гамільтонових систем.

1. Introduction. The concept of resonance in mechanics and physics is defined as follows: a vibrating system excited by a periodic force, whose frequency is equal or close to one natural frequency of vibration of the system, exhibits oscillations of increasing amplitude. According to Richard Feynmann,

If we look in the Physical Reviews, every issue has a resonance curve.

Well known examples are a child pushing another one on a swing, a cristal glass broken by the tune of a singing diva, Jericho's wall falling under the sound of trumpets, a radio or TV antenna and electromagnetic waves, detecting (weak) nuclear magnetism through nuclear magnetic resonance, a bridge in Tours collapsing when crossed by a troup of soldiers which did not break the step, Tacoma bridge exhibiting large oscillations and destruction under the action of the wind, and even the tides: according to Charles Ed. Guillaume,

the ocean is a swing that Moon pushes in cadence.

We first recall the mathematical theory of resonance for a $2 \pi$-periodically forced linear oscillator

$$
\ddot{x}+\lambda x=e(t)
$$

In this case, nonresonance can be characterized by the existence of a (unique) $2 \pi$-periodic, or by the boundedness of all solutions, for all forcings $e(t)$, and resonance can be characterized by the unboundedness of all solutions.

The problem is to see which part of this picture is preserved or destroyed in the case of a boundedly perturbed forced linear oscillator

$$
\ddot{x}+\lambda x=f(x)+e(t)
$$

of a forced asymmetric oscillator

$$
\ddot{x}+\mu x^{+}-\nu x^{-}=e(t),
$$

of a boundedly perturbed forced asymmetric oscillator

$$
\ddot{x}+\mu x^{+}-\nu x^{-}=f(x)+e(t),
$$

of a forced positive homogeneous planar Hamiltonian system

$$
J \dot{u}=\nabla H(u)+p(t),
$$


and of a boundedly perturbed forced positive homogeneous planar Hamiltonian system

$$
J \dot{u}=\nabla H(u)+g(u)+p(t)
$$

Giving answers to those questions require the use of various deep methods of nonlinear analysis and dynamical systems.

2. Forced linear oscillator. 2.1. Free linear oscillator. It is well known that the free linear oscillator

$$
\ddot{x}+\lambda x=0, \quad \lambda>0,
$$

has the solutions

$$
x(t)=A \sin \sqrt{\lambda}(t+\theta), \quad A \geq 0, \quad \theta \in \mathbb{R},
$$

which are all periodic with the same period $2 \pi / \sqrt{\lambda}$.

It follows from the energy integral that, in the phase place $(x, \dot{x})$, the corresponding orbits are given by the family of ellipses centered at $(0,0)$

$$
\frac{\dot{x}^{2}}{2}+\lambda \frac{x^{2}}{2}=E, \quad E \geq 0
$$

so that the origin is an isochronous center with period $2 \pi / \sqrt{\lambda}$.

Given a fixed period, say $2 \pi$, the eigenvalues of (1) are the $\lambda>0$ such that equation (2) has nontrivial $2 \pi$-periodic solutions. They are given by $n^{2}, n \in \mathbb{N}_{0}$.

2.2. Forced linear oscillator: nonresonance. Consider the forced linear oscillator

$$
\ddot{x}+\lambda x=e(t) \quad \lambda>0,
$$

where $e(t)$ is continuous and $2 \pi$-periodic. Its spectrum is the set of $\lambda \in \mathbb{R}$ such that equation (2) has a unique $2 \pi$-periodic solution for each $2 \pi$-periodic continuous $e(t)$. It coincides with the set of eigenvalues of equation (1), because of the following classical elementary result:

Proposition 1. If $\lambda \notin\left\{n^{2}: n \in \mathbb{N}_{0}\right\}$, then, for any continuous $2 \pi$-periodic $e(t)$, equation (2):

1) has a (unique) $2 \pi$-periodic solution;

2) has all its solutions bounded over $\mathbb{R}$ (indeed quasiperiodic).

2.3. Forced linear oscillator: resonance. Any continuous and $2 \pi$-periodic function $e(t)$ has the Fourier expansion

$$
e(t) \sim \frac{e_{0}}{2}+\sum_{n=1}^{\infty}\left(e_{n} \cos n t+f_{n} \sin n t\right)=\sum_{n=-\infty}^{+\infty} \widehat{e}_{n} e^{i n t},
$$

where

$$
\begin{gathered}
e_{n}:=\frac{1}{\pi} \int_{0}^{2 \pi} e(t) \cos n t d t, \quad f_{n}:=\frac{1}{\pi} \int_{0}^{2 \pi} e(t) \sin n t d t \\
\widehat{e}_{n}:=\frac{1}{2 \pi} \int_{0}^{2 \pi} e(t) e^{-i n t} d t
\end{gathered}
$$

ISSN 1027-3190. Укр. мат. журн., 2007, m. 59, № 2 
so that

$$
\widehat{e}_{n}=\overline{\widehat{e}_{-n}}, \quad\left|\widehat{e}_{n}\right|=\left|\widehat{e}_{-n}\right|=\frac{1}{2}\left(e_{n}^{2}+f_{n}^{2}\right)^{1 / 2} .
$$

The following result is also well known:

Proposition 2. All solutions of equation

$$
\ddot{x}+n^{2} x=e(t), \quad n \in \mathbb{N}_{0},
$$

are

1) unbounded if and only if $\left|e_{n}\right|+\left|f_{n}\right| \neq 0$;

2) $2 \pi$-periodic if and only if $\left|e_{n}\right|+\left|f_{n}\right|=0$.

Let us formulate this result in another way which makes more transparent the comparison with further results for nonlinear oscillators. Define the resonance function $\Sigma_{e}^{n}$ for (3) by

$$
\Sigma_{e}^{n}(\theta):=\int_{0}^{2 \pi} e(t) \sin n(t+\theta) d t=\pi\left[f_{n} \cos n \theta+e_{n} \sin n \theta\right], \quad \theta \in \mathbb{R} .
$$

$\Sigma_{e}^{n}$ is $(2 \pi / n)$-periodic, identically zero if and only if $\left|e_{n}\right|+\left|f_{n}\right|=0$, and changes sign (with two simple zeros) in $\left[0,2 \pi / n\right.$ [ if and only if $\left|e_{n}\right|+\left|f_{n}\right| \neq 0$. Hence Proposition 2 trivially implies the following proposition.

Proposition 3. All solutions of equation (3)

1) are unbounded if and only if $\Sigma_{e}^{n}$ changes sign;

2) $2 \pi$-periodic if and only if $\Sigma_{e}^{n} \equiv 0$.

Notice the dual mathematical formulation of the resonance phenomenon for the periodically forced linear oscillator as an alternative between the $2 \pi$-periodic or unbounded character of all responses, depending upon the nature of the forcing term.

Let us finally observe that, for the forced linear operator, either all solutions are bounded over $\mathbb{R}$, or all solutions are unbounded over $\mathbb{R}$.

3. Perturbed forced linear oscillator. 3.1. Nonresonance. We now consider the perturbed forced linear oscillator

$$
\ddot{x}+\lambda x=f(x)+e(t),
$$

where $\lambda>0, f: \mathbb{R} \rightarrow \mathbb{R}$ is continuous, and the unperturbed oscillator is nonresonant.

Theorem 1. If $\lambda \notin\left\{n^{2}: n \in \mathbb{N}_{0}\right\}$ and $f$ is bounded, equation (5) has a $2 \pi$-periodic solution for any continuous and $2 \pi$-periodic forcing $e(t)$.

The proof is a simple consequence of Schauder's fixed point theorem after reduction of the problem to an integral equation using Green's function of the linear part.

So the existence of a $2 \pi$-periodic response survives despite of the nonlinear term, but uniqueness may be lost because of the nonlinear term. For example, the equation

$$
\ddot{x}+(1 / 2) x=\arctan x
$$

has three $2 \pi$-periodic (constant) solutions.

A delicate question in this case is to know if, like in the unperturbed case $(f \equiv$ $\equiv 0$ ), all solutions of (5) are bounded (in $C^{1}$-norm) over $\mathbb{R}$. This is called Littlewood's problem because it was prompted by questions of Littlewood in [32]. The first positive 
answer was given by Morris [40] in 1976 for the superlinear problem $\ddot{x}+2 x^{3}=e(t)$, and many generalizations have been given for this superlinear case. The idea consists in transforming the equation, outside of a large ball of the phase plane $\left(x, x^{\prime}\right)$, into a perturbation of an integrable Hamiltonian system, and to apply Arnold - KolmogorovMoser's twist theorem (see, e.g., [41]) to its Poincaré's map, which is shown to be closed to a twist map outside this ball. The idea was successfully applied by Bin Liu [33] for some class of unbounded perturbations $f$ in equation (5) and, in a subsequent paper [34], to some class of smooth bounded perturbations $f$ and for all smooth $2 \pi$-periodic forcings $e(t)$. See also [30], who assumes $f$ and $e$ smooth and odd, $\lim _{x \rightarrow+\infty} f(x) \neq 0$, some growth conditions at infinity upon the sixth derivative of $f$ and some growth condition upon the first six derivatives of the indefinite integral of $f$.

3.2. Resonance: periodic solutions. The study of the perturbed resonant linear oscillator

$$
\ddot{x}+n^{2} x=f(x)+e(t),
$$

where $n \in \mathbb{N}_{0}$ and $f: \mathbb{R} \rightarrow \mathbb{R}$ is continuous is more delicate. As shown by the unperturbed case, some restriction must be made upon $e(t)$ and $f$.

The following result was proved in 1969 by Lazer and Leach [28]:

Theorem 2. If $f: \mathbb{R} \rightarrow \mathbb{R}$ is bounded and

$$
f( \pm \infty):=\lim _{u \rightarrow \pm \infty} f(u)
$$

exist, equation (6) has a $2 \pi$-periodic solution for each continuous and $2 \pi$-periodic e $(t)$ such that

$$
\left|\widehat{e}_{n}\right|<\frac{1}{\pi}|f(+\infty)-f(-\infty)| .
$$

Lazer - Leach's original proof was based upon a quite involved argument using Lyapunov-Schmidt's decomposition into the subspaces of constant functions and of functions with mean value zero, and Schauder's fixed point theorem. Simpler subsequent proofs have used topological degree arguments (see, e.g., [25]).

Condition (8), nowadays called a Landesman-Lazer condition (and not a LazerLeach condition), because of its (more popular) subsequent version for semilinear elliptic Dirichlet problems, can be written equivalently

$$
\left(e_{n}^{2}+f_{n}^{2}\right)^{1 / 2}<\frac{2}{\pi}|f(+\infty)-f(-\infty)|,
$$

and hence excludes the case where $f \equiv 0$.

Condition (8) is necessary for the class of $f$ such that

$$
\min \{f(-\infty), f(+\infty)\}<f(u)<\max \{f(-\infty), f(+\infty)\}, \quad u \in \mathbb{R} .
$$

For example, equation

$$
\ddot{x}+n^{2} x=a \arctan x+e(t), \quad a \neq 0,
$$

has a $2 \pi$-periodic solution if and only if $\left|\widehat{e}_{n}\right|<|a|$. This shows that the presence of the nonlinearity extends the range of the nonlinear differential operator $\frac{d^{2}}{d t^{2}}+n^{2} I+f(\cdot)$ in 
the space $C_{2 \pi}$ of continuous and $2 \pi$-periodic functions, from the co-dimension two vector subspace $\left\{e \in C_{2 \pi}(\mathbb{R}): \widehat{e_{n}}=0\right\}$, to the open strip $\left\{e \in C_{2 \pi}(\mathbb{R}):\left|\widehat{e_{n}}\right|<|a|\right\}$.

Define the resonance function $\Lambda_{e, f}^{n}$ for (6) by

$$
\Lambda_{f, e}^{n}(\theta):=2[f(+\infty)-f(-\infty)]+\Sigma_{e}^{n}(\theta), \quad \theta \in \mathbb{R} .
$$

Landesman -Lazer's condition can trivially be written in the following equivalent form:

Theorem 3. Equation (6) has a $2 \pi$-periodic solution if its resonance function $\Lambda_{f, e}^{n}$ does not vanish.

Notice that, when $f(+\infty) \neq f(-\infty), \Lambda_{f, e}^{n}$ can have a constant sign for a nonzero $\Sigma_{e}^{n}$.

Again, in the case where a $2 \pi$-periodic solution exists, the question of the boundedness over $\mathbb{R}$ (in $C^{1}$-norm) of all solutions of (6) is a delicate one. Ortega [42] has shown in 1999, using similar techniques as for the nonresonance case, that all solutions are bounded in $\mathbb{R}$ in the $C^{1}$-norm under condition (8) for the special case where $f(u)=-L$ if $x \geq 1$, $-L x$ if $-1 \leq x \leq 1, L$ if $x \leq-1$, and $e$ is of class $C^{5}$. Bin Liu [36] has improved the same year Ortega's result as follows:

Theorem 4. If $f \in C^{6}(\mathbb{R}), \lim _{|x| \rightarrow \infty} x^{6} f^{(6)}(x)=0, e \in C^{7}(\mathbb{R})$, the limits (7) exist, Landesman-Lazer's condition

$$
\left|\widehat{e}_{n}\right|<[f(-\infty)-f(+\infty)]
$$

and inequality

$$
f(+\infty) \leq f(x) \leq f(-\infty), \quad x \in \mathbb{R},
$$

holds, then $\sup _{t \in \mathbb{R}}\left(|x(t)|+\left|x^{\prime}(t)\right|\right)$ for all solutions $x$ of (6).

See also [26].

3.3. Resonance: unbounded solutions. The following nonexistence result for $2 \pi$ periodic solutions of (6) given in the same paper [28] of Lazer and Leach has been less noticed:

Theorem 5. Equation (6) has no $2 \pi$-periodic solution if

$$
\left|\widehat{e}_{n}\right| \geq \frac{1}{\pi}\left[\sup _{\mathbb{R}} f-\inf _{\mathbb{R}} f\right]>0 .
$$

Inequality (10) looks like a negation of Landesman-Lazer condition (8), except that $\sup _{\mathbb{R}} f$ and $\inf _{\mathbb{R}} f$ replace the limits of $f$ at $\pm \infty$.

Let us now recall a classical result of Massera [39]:

Lemma 1. Equation

$$
\ddot{x}=h(t, x, \dot{x})
$$

with $h 2 \pi$-periodic in $t$, has a $2 \pi$-periodic solution if its Cauchy problem is uniquely globally solvable, and if it has a solution bounded in the future or in the past.

Combining Theorem 5 with Lemma 1, we obtain the following result, which can essentially be traced to Seifert [48]:

Corollary 1. Equation (6) with $f$ locally Lipschitzian has all solutions unbounded in $]-\infty, 0]$ and in $[0,+\infty[$ if condition (10) holds.

Condition (10) can be sharpened when $f( \pm \infty)$ exist in a generalized sense introduced in 1996 by Alonso-Ortega [1]. For $f: \mathbb{R} \rightarrow \mathbb{R}$ continuous and bounded, define 


$$
F(s):=\int_{0}^{s} f(v) d v, \quad F^{ \pm}:=\lim _{s \rightarrow \pm \infty} \frac{F(s)}{s},
$$

when those limits exist. Using l'Hospital's rule, it is easy to see that $F^{ \pm}=f( \pm \infty)$ if $f( \pm \infty)$ exist, but, for $f(s)=a \arctan s+b \sin s, F^{ \pm}= \pm \frac{a \pi}{2}$ although $f( \pm \infty)$ do not exist.

When those generalized limits exist, the resonance function $\Omega_{f, e}^{n}$ for (6) is defined by

$$
\Omega_{f, e}^{n}(\theta):=2\left[F^{+}-F^{-}\right]+\Sigma_{e}^{n}(\theta), \quad \theta \in \mathbb{R},
$$

and reduces to $\Lambda_{f, e}^{n}$ when $f( \pm \infty)$ exist.

Alonso and Ortega [1] have generalized as follows Corollary 1.

Theorem 6. If $f$ is locally Lipschitzian and $\Omega_{f, e}^{n}(\theta)$ changes sign, there exists $R>$ $>0$ such that for any solution of (6) with $x^{2}(0)+\dot{x}^{2}(0)>R$, one has either

$$
\lim _{t \rightarrow+\infty}\left[x^{2}(t)+\dot{x}^{2}(t)\right]=+\infty
$$

or

$$
\lim _{t \rightarrow-\infty}\left[x^{2}(t)+\dot{x}^{2}(t)\right]=+\infty .
$$

The proof uses properties of Poincaré's map which associates to each $\left(x_{0}, \dot{x}_{0}\right) \in \mathbb{R}^{2}$ the point $\left(x\left(2 \pi ; x_{0}, \dot{x}_{0}\right), \dot{x}\left(2 \pi ; x_{0}, \dot{x}_{0}\right)\right)$, where $x\left(t ; x_{0}, \dot{x}_{0}\right)$ denotes the solution of (6) with initial conditions $x_{0}$ and $\dot{x}_{0}$.

The assumption upon $\Omega_{f, e}^{n}$ in Theorem 6 is of course equivalent to

$$
\left|\widehat{e}_{n}\right|>\frac{1}{\pi}\left|F^{+}-F^{-}\right|
$$

The example

$$
\ddot{x}+x=-\sin x+\sin (\sin t)
$$

which satisfies the conditions of Theorem 6 with $n=1$ and has the $2 \pi$-periodic solution $x(t)=\sin t$ shows that, in contrast to the linear case, $2 \pi$-periodic and unbounded solutions may coexist in a perturbed resonant oscillator.

4. Forced asymmetric oscillator. 4.1. Free asymmetric oscillator. The free linear oscillator can be generalized to the free asymmetric oscillator

$$
\ddot{x}+\mu x^{+}-\nu x^{-}=0, \quad \mu>0, \quad \nu>0,
$$

where $x^{+}:=\max (x, 0), x^{-}:=\max (-x, 0)$. The linear restoring force is replaced by a piecewise linear one.

It easily follows from the energy integral

$$
\frac{\dot{x}^{2}}{2}+\mu \frac{\left(x^{+}\right)^{2}}{2}+\nu \frac{\left(x^{-}\right)^{2}}{2}=E, \quad E \geq 0,
$$

that the orbits of (12) in the phase plane $(x, \dot{x})$ are egg-shaped closed curves around the origin made by half-ellipses of equation

$$
\frac{\dot{x}^{2}}{2}+\mu \frac{x^{2}}{2}=E
$$

ISSN 1027-3190. Укр. мат. журн., 2007, m. 59, № 2 
in the right-hand half plane and

$$
\frac{\dot{x}^{2}}{2}+\nu \frac{x^{2}}{2}=E
$$

in the left-hand half plane. Hence, the origin is an isochronous center with period $\pi\left(\frac{1}{\sqrt{\mu}}+\right.$ $\left.+\frac{1}{\sqrt{\nu}}\right)$

A generalized concept of eigenvalue has been introduced by Fučik [23] and by Dancer [12] for the asymmetric oscillator with various boundary conditions. A Fučik's eigenvalue for (12) is any $(\mu, \nu) \in \mathbb{R}^{2}$ such that equation (12) has a nontrivial $2 \pi$-periodic solution. It is easy to see that the set $\sigma_{F}$ of positive Fučik eigenvalues is given by the following family of hyperbolic-like curves (Fučik's curves)

$$
\bigcup_{n \in \mathbb{N}_{0}}\left\{(\mu, \nu) \in \mathbb{R}_{+}^{2}: \frac{1}{\sqrt{\mu}}+\frac{1}{\sqrt{\nu}}=\frac{2}{n}\right\}:=\bigcup_{n \in \mathbb{N}_{0}} \sigma_{F}^{n} .
$$

Notice that $\sigma_{F}^{n}$ intersects the diagonal in the $(\mu, \nu)$-plane at the point $\left(n^{2}, n^{2}\right)$. If one defines the corresponding Fučik's eigenfunctions by

$$
\sigma_{n}(t):= \begin{cases}\frac{1}{\sqrt{\mu}} \sin (\sqrt{\mu} t) & \text { for } t \in\left[0, \frac{\pi}{\sqrt{\mu}}\right], \\ -\frac{1}{\sqrt{\nu}} \sin \left(\sqrt{\nu}\left(t-\frac{\pi}{\sqrt{\mu}}\right)\right) & \text { for } t \in\left[\frac{\pi}{\sqrt{\mu}}, \frac{2 \pi}{n}\right],\end{cases}
$$

the nontrivial solutions of (12) are given by

$$
x(t)=A \sigma_{n}(t+\theta), \quad A>0, \quad \theta \in \mathbb{R},
$$

and are $(2 \pi / n)$-periodic.

4.2. Nonresonance: periodic solutions. Consider now the forced asymmetric oscillator

$$
\ddot{x}+\mu x^{+}-\nu x^{-}=e(t), \quad \mu>0, \quad \nu>0 .
$$

The following result is essentially due to Fučik [23]:

Theorem 7. If $(\mu, \nu) \notin \sigma_{F}$, equation (13) has a $2 \pi$-periodic solution for any $e(t)$.

The proof of the first part is based upon Leray-Schauder degree, using a homotopy which deforms (13) into a linear problem of the form $\ddot{x}+\lambda x=0$, with $(\lambda, \lambda)$ located between the same two Fučik curves as $(\mu, \nu)$. It is also a trivial consequence of Corollary 8 in [8].

When $(\mu, \nu) \in \sigma_{F}^{n}$ for some $n \in \mathbb{N}_{0}$, Dancer [12] has given an example of $e(t)$ such that equation (13) has no $2 \pi$-periodic solution.

Because of this result, the set $\sigma_{F}$ is also called the positive Fučik's spectrum of (12).

The boundedness over $\mathbb{R}$ of all solutions of (13) has been also considered. Ortega [42] has shown in 1993 that (13) has all its solutions bounded (in $C^{1}$-norm) over $\mathbb{R}$ if $e(t)=1+p(t)$ is of class $C^{4}$ and $p$ is sufficiently small in $C^{4}$-norm. 
4.3. Resonance: periodic solutions. If $(\mu, \nu) \in \sigma_{F}^{n}$ for some $n \in \mathbb{N}_{0}$, Dancer [12] has introduced in 1977 the resonance function $\Delta_{e}^{n}$ for (13) defined by

$$
\Delta_{e}^{n}(\theta):=n \int_{0}^{2 \pi} e(t) \sigma_{n}(t+\theta) d t, \quad \theta \in \mathbb{R} .
$$

$\Delta_{e}^{n}$ is $(2 \pi / n)$-periodic and reduces to $\Sigma_{e}^{n}$ for $\mu=\nu=n^{2}$. Because of the richer harmonic structure of $\sigma(t)$ with respect to $\sin n t, \Delta_{e}^{n}$ may have an arbitrary large (even) number of zeros in $[0,2 \pi / n[$.

The following existence result for $2 \pi$-periodic solutions of a forced resonant asymmetric oscillator is due to Dancer [12].

Theorem 8. If $(\mu, \nu) \in \sigma_{F}^{n}$ for some $n \in \mathbb{N}_{0}$, and $\Delta_{e}^{n}$ does not vanish, equation (13) has a $2 \pi$-periodic solution.

The proof is based upon some perturbation technique and homotopy to a linear problem with Leray - Schauder degree one.

A further existence condition has been obtained in 1998 by Fabry and Fonda [15].

Theorem 9. If $(\mu, \nu) \in \sigma_{F}^{n}$ for some $n \in \mathbb{N}_{0}$, and $\Delta_{e}^{n}$ has more than two zeros in $[0,2 \pi / n[$, all simple, equation (13) has a $2 \pi$-periodic solution.

The proof is based upon the fact that Brouwer's degree of the associated Poincaré's map minus identity on large balls is shown to be equal to $1-z$, with $2 z$ the number of zeros of $\Delta_{e}^{n}$ in $[0,2 \pi / n[$.

For further results see [57].

We will see in the next section that unbouded solutions exist when $\Delta_{e}^{n}$ changes sign. The question of boundedness over $\mathbb{R}$ of all solutions when $\Delta_{e}^{n}$ does not vanish has been considered by in 1999 by Bin Liu [35], who has proved, using twist maps techniques, the following result:

Theorem 10. If e is of class $C^{6},(\mu, \nu) \in \sigma_{F}^{n}$ for some $n \in \mathbb{N}_{0}$, and $\Delta_{e}^{n}$ does not vanish, then all solutions of (13) are bounded over $\mathbb{R}$ in $C^{1}$-norm.

A similar result holds when

$$
\frac{1}{\sqrt{\mu}}+\frac{1}{\sqrt{\nu}}=2 \frac{m}{n}
$$

for some relativitely prime positive integers. For related results, see [5].

4.4. Resonance: unbounded solutions. Alonso and Ortega [2] have obtained in 1998 the following sufficient condition for the existence of unbounded solutions.

Theorem 11. Let $(\mu, \nu) \in \sigma_{F}^{n}$ for some $n \in \mathbb{N}_{0}$. If $\Delta_{e}^{n}$ has zeros, all simple, there exists $R>0$ such that for any solution $x(t)$ of (13) with $x^{2}\left(t_{0}\right)+\dot{x}^{2}\left(t_{0}\right)>R$ for some $t_{0} \in \mathbb{R}$, one has either

$$
\lim _{t \rightarrow+\infty}\left[x^{2}(t)+\dot{x}^{2}(t)\right]=+\infty
$$

or

$$
\lim _{t \rightarrow-\infty}\left[x^{2}(t)+\dot{x}^{2}(t)\right]=+\infty
$$

The proof is based on study of the dynamics of a class of planar mappings associated to Poincaré's map. 
Alonso and Ortega have also shown that $2 \pi$-periodic and unbounded solutions may coexist in (13), e.g. for infinitely many $r$ in equation

$$
\ddot{x}+\mu x^{+}-\nu x^{-}=\cos r t .
$$

For further results see $[55,56]$.

5. Perturbed forced asymmetric oscillator. 5.1. Nonresonance. Consider the perturbed forced asymmetric oscillator

$$
\ddot{x}+\mu x^{+}-\nu x^{-}=f(x)+e(t), \quad \mu>0, \quad \nu>0,
$$

where $f: \mathbb{R} \rightarrow \mathbb{R}$ is continuous and $e(t)$ is continuous and $2 \pi$-periodic.

Theorem 12. If $(\mu, \nu) \notin \sigma_{F}$ and $f$ is bounded, equation (15) has a $2 \pi$-periodic solution for any $e(t)$.

The proof of this result is an application of Leray - Schauder degree. For example, it is a consequence of Corollary 8 in [8].

So Fučik's existence result for $g \equiv 0$ survives despite of the nonlinear perturbation $g$.

5.2. Resonance: periodic solutions. In 2000, Fabry and Mawhin [18] have extended Landesman - Lazer's and Fabry - Fonda's existence conditions to the resonant perturbed forced asymmetric oscillator, in terms of the resonance function $\Psi_{f, e}^{n}$ for (15) defined by

$$
\Psi_{f, e}^{n}(\theta):=2 n^{2}\left[\frac{F^{+}}{\mu}-\frac{F^{-}}{\nu}\right]+\Delta_{e}^{n}(\theta), \quad \theta \in \mathbb{R},
$$

where $F^{ \pm}$is defined in (11). $\Psi_{f, e}^{n}$ is $2 \pi / n$-periodic and reduces to $\Omega_{f, e}^{n}$ for $\mu=\nu=n^{2}$.

Theorem 13. If $(\mu, \nu) \in \sigma_{F}^{n}$ for some $n \in \mathbb{N}_{0}, f$ is bounded and locally Lipschitzian, $F^{ \pm}$exist, and $\Psi_{f, e}^{n}$ has no zero or more than two zeros in $\left[0, \frac{2 \pi}{n}[\right.$, all simple, then equation (15) has a $2 \pi$-periodic solution.

The proof is based upon the fact that if $2 z$ denotes the number of zeros of $\Psi_{f, e}^{n}$, the Brouwer degree of Poincaré's map minus identity is shown to be equal to $1-z$.

A basic ingredient for this proof and the one of the results of next subsection is the use of the averaging method [3] to compare the solutions of large amplitude of (15) written in the equivalent form

$$
\begin{gathered}
\dot{\rho}_{\epsilon}=\epsilon f\left(\frac{\rho_{\epsilon}}{\epsilon} \sigma_{n}(t+\theta)\right) \dot{\sigma}_{n}(t+\theta)+\epsilon e(t) \dot{\sigma_{n}}(t+\theta), \\
\dot{\theta}=-\frac{\epsilon}{\rho_{\epsilon}} f\left(\frac{\rho_{\epsilon}}{\epsilon} \sigma_{n}(t+\theta)\right) \sigma_{n}(t+\theta)-\frac{\epsilon}{\rho_{\epsilon}} e(t) \sigma_{n}(t+\theta),
\end{gathered}
$$

through the change of unknowns

$$
x(t)=\frac{\rho_{\epsilon}(t)}{\epsilon} \sigma_{n}(t+\theta(t)), \quad \dot{x}(t)=\frac{\rho_{\epsilon}(t)}{\epsilon} \dot{\sigma_{n}}(t+\theta(t)),
$$

to the averaged equation over $[0,2 \pi]$ of an asymptotic version of system (17), which happens to be

$$
\dot{\tilde{\rho}}=\frac{\epsilon}{2 n \pi}\left(\Psi_{f, e}^{n}\right)^{\prime}(\widetilde{\theta}), \quad \dot{\tilde{\theta}}=-\frac{\epsilon}{2 n \pi \widetilde{\rho}} \Psi_{f, e}^{n}(\widetilde{\theta})
$$

For further related results, see $[11,49,4,6]$. 
The existence of infinitely many subharmonic solutions has also been obtained in [18]. For other results based upon twist maps techniques, see [50,51,7]. The boundedness of all solutions of equation (15) has been recently considered by Xiaojing Yang [60].

5.3. Resonance: unbounded solutions. Fabry and Mawhin [18] have also extended Alonso - Ortega's result about unbounded solutions to the resonant perturbed forced asymmetric oscillator.

Theorem 14. If $(\mu, \nu) \in \sigma_{F}^{n}$ for some $n \in \mathbb{N}_{0}, f$ is bounded and locally Lipschitzian, $F^{ \pm}$exist, and $\Psi_{f, e}^{n}$ has zeros in $\left[0, \frac{2 \pi}{n}[\right.$, all simple, any solution $x(t)=$ $=\rho(t) \sigma_{n}(t+\theta(t))$ of (15) with $\rho(0)$ sufficiently large is unbounded either in the past or in the future.

The proof is a delicate application of the averaging method indicated above, together with Riemann - Lebesgue type theorems for oscillatory integrals.

6. Forced planar Hamiltonian systems. 6.1. Free planar Hamiltonian systems. The free linear and asymmetric oscillators are special cases of free planar Hamiltonian systems of the form

$$
J \dot{u}=\nabla \mathcal{H}(u)
$$

where $J=\left(\begin{array}{cc}0 & -1 \\ 1 & 0\end{array}\right), \mathcal{H}: \mathbb{R}^{2} \rightarrow \mathbb{R}$ is positive and positively homogeneous of degree two, and $\nabla \mathcal{H}: \mathbb{R}^{2} \rightarrow \mathbb{R}^{2}$ is continuous.

Because of the energy integral $\mathcal{H}(u)=E(E \geq 0)$, the orbits of the solutions of (18) in the phase plane $\mathbb{R}^{2}$ are closed curves surrounding the origin, and, because of the positive homogeneity of degree two of $\mathcal{H}$, the corresponding solutions all have the same minimal period $\tau$. Hence the origin is an isochronous center.

6.2. Nonresonance: periodic solutions. We now consider the forced planar Hamiltonian system

$$
J \dot{u}=\nabla \mathcal{H}(u)+p(t),
$$

where $J$ and $\mathcal{H}$ are like above and $p: \mathbb{R} \rightarrow \mathbb{R}^{2}$ is $2 \pi$-periodic and continuous.

The following results were proved by Fonda [20] in 2004.

Theorem 15. If $\mathcal{H}: \mathbb{R}^{2} \rightarrow \mathbb{R}$ is positive and positively homogeneous of degree two, $\nabla \mathcal{H}: \mathbb{R}^{2} \rightarrow \mathbb{R}^{2}$ is continuous, and $\frac{2 \pi}{\tau} \notin \mathbb{N}$, system (19) has a $2 \pi$-periodic solution for any $p(t)$.

The proof uses Corollary 8 of [8].

Theorem 16. If $\mathcal{H}: \mathbb{R}^{2} \rightarrow \mathbb{R}$ is positive and positively homogeneous of degree two, $\nabla \mathcal{H}: \mathbb{R}^{2} \rightarrow \mathbb{R}^{2}$ is locally Lipschitzian and $\frac{2 \pi}{\tau} \notin \mathbb{N}$, there exists $p(t)$ for which all solutions of system (19) are unbounded, namely $\lim _{t \rightarrow+\infty}\|u(t)\|=+\infty$.

The proof consists in constructing such a function $p$ by a technique generalizing examples of Dancer [13] and of Ortega [47] for second order equations.

6.3. Resonance: periodic solutions. When $\frac{2 \pi}{\tau}=n \in \mathbb{N}_{0}$, Fonda [20] has introduced in 2004 the resonance function $\Phi_{p}^{n}: \mathbb{R}^{2} \rightarrow \mathbb{R}$ of (19) defined by

$$
\Phi_{p}^{n}(\theta)=\int_{0}^{2 \pi}\left\langle p(t) \mid \varphi^{n}(t+\theta)\right\rangle d t, \quad \theta \in \mathbb{R}
$$


where $\varphi^{n}$ is a fixed solution of (18) such that $H\left(\varphi^{n}(t)\right)=\frac{1}{2}$. Notice that $\Phi_{p}^{n}$ is $(2 \pi / n)$ periodic.

Fonda [20] has also proved the following result.

Theorem 17. If $\mathcal{H}: \mathbb{R}^{2} \rightarrow \mathbb{R}$ is positive and positively homogeneous of degree two, $\nabla \mathcal{H}: \mathbb{R}^{2} \rightarrow \mathbb{R}^{2}$ is locally Lipschitzian, $\frac{2 \pi}{\tau}=n \in \mathbb{N}_{0}$, and $\Phi_{p}^{n}(\theta)$ never changes sign or has at least four zeros in $[0, \tau[$, all simple, then system (19) has a $2 \pi$-periodic solution.

6.4. Resonance: unbounded solutions. The existence of unbounded solutions has also been proved by Fonda [20].

Theorem 18. If $\mathcal{H}: \mathbb{R}^{2} \rightarrow \mathbb{R}$ is positive and positively homogeneous of degree two, $\nabla \mathcal{H}: \mathbb{R}^{2} \rightarrow \mathbb{R}^{2}$ is locally Lipschitzian, $\frac{2 \pi}{\tau}=n \in \mathbb{N}_{0}$, and $\Phi_{p}^{n}(\theta)$ has zeros in $[0, \tau[$, all simple, then all solutions of system (19) with sufficiently large amplitude are unbounded, either in the past, or in the future.

The proof applies a result of Alonso-Ortega [2] to the equivalent system written in generalized polar coordinates associated to the solutions of (18).

Comparing Theorems 17 and 18, one sees that, again, periodic and unbounded solutions may coexist.

7. Perturbed forced planar Hamiltonian systems. 7.1. Nonresonance. We now consider the perturbed forced planar Hamiltonian system

$$
J \dot{u}=\nabla \mathcal{H}(u)+g(u)+p(t)
$$

where $g: \mathbb{R}^{2} \rightarrow \mathbb{R}^{2}$ is continuous and $p: \mathbb{R} \rightarrow \mathbb{R}^{2}$ is continuous and $2 \pi$-periodic.

Theorem 19. If $2 \pi / \tau \notin \mathbb{N}_{0}, \mathcal{H}: \mathbb{R}^{2} \rightarrow \mathbb{R}$ is of class $C^{1}$, positive and positively homogeneous of degree two, $g: \mathbb{R}^{2} \rightarrow \mathbb{R}^{2}$ is bounded, then system (21) has a $2 \pi$-peridoic solution for any $p(t)$.

The proof uses Corollary 8 of [8].

7.2. Resonance: periodic solutions. In order to generalize Landesman-Lazer's and Fabry - Fonda's conditions to system (21), Fonda and Mawhin [22] have introduced in 2006 the following class of bounded nonlinear perturbations $g$, which extends, in our setting, systems of Lur'e type in control theory [38], and contains, as we will see, the special case of second order differential equations with separated nonlinearities.

Assume that there exist directions

$$
0 \leq \vartheta_{1}<\vartheta_{2}<\ldots<\vartheta_{m}<2 \pi
$$

and locally Lipschitzian functions $g_{k}: \mathbb{R} \rightarrow \mathbb{R}^{2}$ such that

$$
g(u)=\sum_{k=1}^{m} g_{k}\left(\left\langle u \mid e^{i \vartheta_{k}}\right\rangle\right)
$$

If $\tau=\frac{2 \pi}{n}$ for some $n \in \mathbb{N}_{0}$, and if

$$
G_{k}(x):=\int_{0}^{x} g_{k}(s) d s, \quad G_{k}^{ \pm}:=\lim _{s \rightarrow \pm \infty} \frac{G_{k}(s)}{s}, \quad 1 \leq k \leq m,
$$

exist, Fonda and Mawhin have defined as follows the resonance mapping $\Gamma^{g, p}: \mathbb{R}^{2} \rightarrow \mathbb{R}^{2}$ for (21). Let 


$$
\begin{gathered}
\mathcal{A}_{k}^{+}:=\left\{t \in[0, \tau]:\left\langle\varphi^{n}(t) \mid e^{i \vartheta_{k}}\right\rangle>0\right\}, \\
\mathcal{A}_{k}^{-}:=\left\{t \in[0, \tau]:\left\langle\varphi^{n}(t) \mid e^{i \vartheta_{k}}\right\rangle<0\right\}, \\
\kappa_{1}^{g}:=n \sum_{k=1}^{m}\left(\left\langle G_{k}^{+} \mid \int_{\mathcal{A}_{k}^{+}} \varphi^{n}\right\rangle+\left\langle G_{k}^{-} \mid \int_{\mathcal{A}_{k}^{-}} \varphi^{n}\right\rangle\right), \\
\kappa_{2}^{g}:=n \sum_{k=1}^{m}\left(\left\langle G_{k}^{+} \mid \int_{\mathcal{A}_{k}^{+}} \dot{\varphi}^{n}\right\rangle+\left\langle G_{k}^{-} \mid \int_{\mathcal{A}_{k}^{-}} \dot{\varphi}^{n}\right\rangle\right),
\end{gathered}
$$

where $\varphi^{n}$ is, like above, a fixed solution of (18) such that $H\left(\varphi^{n}(t)\right)=\frac{1}{2}$. Then $\Gamma^{g, p}$ is defined by

$$
\Gamma_{1}^{g, p}(\theta):=-\kappa_{1}^{g}-\Phi_{p}^{n}(\theta), \quad \Gamma_{2}^{g, p}(\theta):=\kappa_{2}^{g}+\left(\Phi_{p}^{n}\right)^{\prime}(\theta) .
$$

In the special case of equation

$$
\ddot{x}+\mu x^{+}-\nu x^{-}=f(x)+e(t)
$$

written as a Hamiltonian system, $\Gamma^{f, e}$ reduces to

$$
\left(\Psi_{f, e}^{n},-\left(\Psi_{e}^{n}\right)^{\prime}\right)=\left(\Psi_{f, e}^{n},-\left(\Delta_{e}^{n}\right)^{\prime}\right) .
$$

The following results of Landesman - Lazer's and Fabry - Fonda's types hold.

Theorem 20. If $\mathcal{H}: \mathbb{R}^{2} \rightarrow \mathbb{R}$ is positive and positively homogeneous of degree two, $\nabla \mathcal{H}: \mathbb{R}^{2} \rightarrow \mathbb{R}^{2}$ is locally Lipschitzian, $\frac{2 \pi}{\tau}=n \in \mathbb{N}_{0}, g: \mathbb{R}^{2} \rightarrow \mathbb{R}^{2}$ is locally Lipschitzian, bounded, of type (22), the sets

$$
\left\{u \in \mathbb{R}^{2}:\|u\|=1 \quad \text { and } \quad \frac{\nabla \mathcal{H}(u)}{\|\nabla \mathcal{H}(u)\|}= \pm e^{i \vartheta_{k}}\right\}, \quad 1 \leq k \leq m,
$$

have only isolated points, limits (23) exist, and if

1) either $\Gamma_{1}^{g, p}$ has constant sign or

2) $\Gamma^{g, p}$ does not vanish and either:

a) $\Gamma_{2}^{g, p}$ has constant sign or

b) $\Gamma_{2}^{g, p}$ changes sign more than twice on the zeros of $\Gamma_{1}^{g, p}$,

then system (21) has a $2 \pi$-periodic solution.

Like in paper [18], the joint main ingredient of the proof of this result and of next one is the following. By the change of variable $v=\delta u$, for some $\delta>0$, equation (21) becomes

$$
J \dot{v}=\nabla \mathcal{H}(v)+\delta g\left(\frac{v}{\delta}\right)+\delta p(t) .
$$

If $v(t)$ is a solution of (25) with starting point $v(0) \neq 0$, we can write

$$
v(t)=r(t) \varphi^{n}(t+\theta(t))
$$

with $r(0)>0$. As long as $r(t)>0$, the functions $\theta(t)$ and $r(t)$ are of class $C^{1}$ and satisfy

$$
\theta^{\prime}=\frac{\delta}{r}\left\langle g\left(\frac{r}{\delta} \varphi^{n}(t+\theta)\right)+p(t) \mid \varphi^{n}(t+\theta)\right\rangle,
$$

ISSN 1027-3190. Укр. мат. журн., 2007, m. 59, № 2 


$$
r^{\prime}=-\delta\left\langle g\left(\frac{r}{\delta} \varphi^{n}(t+\theta)\right)+p(t) \mid \dot{\varphi}^{n}(t+\theta)\right\rangle
$$

Denote by $\left(\theta\left(t ; \theta_{0}, r_{0} ; \delta\right), r\left(t ; \theta_{0}, r_{0} ; \delta\right)\right)$ the solution of $(26)$ with starting point

$$
\theta\left(0 ; \theta_{0}, r_{0} ; \delta\right)=\theta_{0} \in\left[0, \tau\left[, \quad r\left(0 ; \theta_{0}, r_{0} ; \delta\right)=r_{0}>0\right.\right.
$$

For $\delta$ small enough, writing $\theta(t)$ for $\theta\left(t ; \theta_{0}, r_{0} ; \delta\right)$, and $r(t)$ for $r\left(t ; \theta_{0}, r_{0} ; \delta\right)$, and setting $\theta_{1}=\theta\left(2 \pi ; \theta_{0}, r_{0} ; \delta\right), r_{1}=r\left(2 \pi ; \theta_{0}, r_{0} ; \delta\right)$, we have

$$
\begin{gathered}
\theta_{1}=\theta_{0}+\delta \int_{0}^{T} \frac{1}{r(t)}\left\langle f\left(\frac{r(t)}{\delta} \varphi(t+\theta(t))\right)+p(t) \mid \varphi(t+\theta(t))\right\rangle d t \\
r_{1}=r_{0}-\delta \int_{0}^{T}\left\langle f\left(\frac{r(t)}{\delta} \varphi(t+\theta(t))\right)+p(t) \mid \dot{\varphi}(t+\theta(t))\right\rangle d t
\end{gathered}
$$

Through a detailed study of the involved oscillatory integrals, one proves the following lemma.

Lemma 2. We have

$$
\begin{gathered}
\theta_{1}=\theta_{0}-\frac{\delta}{r_{0}}\left[\Gamma_{1}\left(\theta_{0}\right)+R_{1}\left(\theta_{0}, r_{0} ; \delta\right)\right] \\
r_{1}=r_{0}-\delta\left[\Gamma_{2}\left(\theta_{0}\right)+R_{2}\left(\theta_{0}, r_{0} ; \delta\right)\right]
\end{gathered}
$$

where $R_{1}$ and $R_{2}$ are such that

$$
\lim _{\delta \rightarrow 0^{+}} R_{1}\left(\theta_{0}, r_{0} ; \delta\right)=\lim _{\delta \rightarrow 0^{+}} R_{2}\left(\theta_{0}, r_{0} ; \delta\right)=0
$$

uniformly for $\theta_{0} \in[0, \tau]$ and $r_{0}$ in a compact subset of $\mathbb{R}^{+}$.

The proof of Theorem 20 is a combination of Lemma 2 and of a formula for the computation of the Brouwer degree of Poincaré's map minus identity on large balls in terms of the properties of $\Gamma^{g, p}$.

For other results, see [17, 21, 59].

7.3. Resonance: unbounded solutions. The following existence results for unbounded solutions are proved in [22].

Theorem 21. If $\mathcal{H}: \mathbb{R}^{2} \rightarrow \mathbb{R}$ is positive and positively homogeneous of degree two, $\nabla \mathcal{H}: \mathbb{R}^{2} \rightarrow \mathbb{R}^{2}$ is locally Lipschitzian, $\frac{2 \pi}{\tau}=n \in \mathbb{N}_{0}, g: \mathbb{R}^{2} \rightarrow \mathbb{R}^{2}$ is locally Lipschitzian, bounded, of type (22), the sets

$$
\left\{u \in \mathbb{R}^{2}:\|u\|=1 \quad \text { and } \quad \frac{\nabla \mathcal{H}(u)}{\|\nabla \mathcal{H}(u)\|}= \pm e^{i \vartheta_{k}}\right\}, \quad 1 \leq k \leq m
$$

have only isolated points, limits (23) exist, and $\Gamma_{1}^{g, p}$ is of constant sign, then:

1) if $\kappa_{2}^{g}>0$, all solutions of system (21) are bounded in the future, and those with sufficiently large amplitude are unbounded in the past;

2) if $\kappa_{2}^{g}<0$, all solutions of system (21) are bounded in the past, and those with sufficiently large amplitudes are unbounded in the future. 
Theorem 22. If $\mathcal{H}: \mathbb{R}^{2} \rightarrow \mathbb{R}$ is positive and positively homogeneous of degree two, $\nabla \mathcal{H}: \mathbb{R}^{2} \rightarrow \mathbb{R}^{2}$ is locally Lipschitzian, $\frac{2 \pi}{\tau}=n \in \mathbb{N}_{0}, g: \mathbb{R}^{2} \rightarrow \mathbb{R}^{2}$ is locally Lipschitzian, bounded, of type (22), the sets

$$
\left\{u \in \mathbb{R}^{2}:\|u\|=1 \quad \text { and } \quad \frac{\nabla \mathcal{H}(u)}{\|\nabla \mathcal{H}(u)\|}= \pm e^{i \vartheta_{k}}\right\}, \quad 1 \leq k \leq m,
$$

have only isolated points, limits (23) exist, and if

1) $\Gamma^{g, p}$ does not vanish;

2) $\Gamma_{1}^{g, p}(\theta)$ changes sign, only having simple zeros,

then all solutions of system (21) with sufficiently large amplitude are unbounded, either in the future, or in the past.

The proofs of those theorems are based upon some results of [17].

For other statements, see [17].

7.4. Applications to second order differential equations. Those theorems can be applied to second order differential equations with separated nonlinearities of the form

$$
\ddot{x}+b(x) \dot{x}+\mu x^{+}-\nu x^{-}+a(x)=e(t)
$$

(Liénard equations),

$$
\ddot{x}+c(\dot{x})+\mu x^{+}-\nu x^{-}+a(x)=e(t)
$$

(Rayleigh equations) or to second order differential equations with mixed nonlinearities of the form

$$
\ddot{x}+\mu x^{+}-\nu x^{-}+a(x+c \dot{x})=e(t) .
$$

For Liénard equations, the obtained existence conditions depend upon

$$
\begin{aligned}
& \lim _{x \rightarrow \pm \infty} \frac{A(x)}{x}:=A^{ \pm}, \\
& \lim _{x \rightarrow \pm \infty} \frac{\mathcal{B}(x)}{x}:=\mathcal{B}^{ \pm},
\end{aligned}
$$

where

$$
A(x):=\int_{0}^{x} a(s) d s, \quad \mathcal{B}(x)=\int_{0}^{x} \ldots\left(\int_{0}^{s} \ldots b(\sigma) d \sigma\right) d s .
$$

For related work about second order equations with separated nonlinearities, see [9, $10,27,29,30,37,31,52,53,58]$.

1. Alonso J. M., Ortega R. Unbounded solutions of semilinear equations at resonance // Nonlinearity. -1996. -9. - P. $1099-1111$

2. Alonso J. M., Ortega R. Roots of unity and unbounded motions of an asymmetric oscillator // J. Different. Equat. - 1998. - 143. - P. 201-220.

3. Bogolyubov N. N., Mitropolskii Yu. A. Asymptotic methods in the theory of nonlinear oscillations. - New York: Gordon and Breach, 1961.

4. Bonheure D., Fabry C. Unbounded solutions of forced isochronous oscillators at resonance // Different. Integr. Equat. - 2002. - 15. - P. $1139-1152$

5. Bonheure D., Fabry C. Littlewood's problem for isochronous oscillations (to appear). 
6. Bonheure D., Fabry C., Smets D. Periodic solutions of forced isochronous oscillators at resonance // Discrete Contin. Dynam. Systems. - 2002. - 8. - P. 907 - 930.

7. Capietto A., Bin Liu. Quasi-periodic solutions of a forced asymmetric oscillator at resonance (to appear).

8. Capietto A., Mawhin J., Zanolin F. Continuation theorems for periodic perturbations of autonomous systems // Trans. Amer. Math. Soc. - 1992. - 329. - P. 41 - 72.

9. Capietto A., Zaihong Wang. Periodic solutions of Liénard equations at resonance // Different. Integr. Equat. - 2003. - 16. - P. 605-624.

10. Capietto A., Zaihong Wang. Periodic solutions of Liénard equations with asymmetric nonlinearities at resonance // J. London Math. Soc. - 2003. - 68. - P. 119-132.

11. Dambrosio $W$. A note on the existence of unbounded solutions to a perturbed asymmetric oscillator // Nonlinear Anal. - 2002. - 50. - P. 333 - 346.

12. Dancer E. N. Boundary-value problems for weakly nonlinear ordinary differential equations // Bull. Austral. Math. Soc. - 1976. - 15. - P. $321-328$

13. Dancer E. N. Proofs of the results of 'boundary-value problems for weakly nonlinear ordinary differential equations' (unpublished).

14. Fabry $C$. Landesman - Lazer conditions for periodic boundary value problems with asymmetric nonlinearities // J. Different. Equat. - 1995. - 116. - P. 405-418.

15. Fabry C., Fonda A. Nonlinear resonance in asymmetric oscillators // Ibid. - 1998. - 147. - P. 58 - 78.

16. Fabry C., Fonda A. Periodic solutions of perturbed isochronous Hamiltonian systems at resonance // Ibid. - 2005. - 214. - P. $299-325$.

17. Fabry C., Fonda A. Unbounded motions of perturbed isochronous Hamiltonian systems at resonance // Adv. Nonlinear Stud. - 2005. - 5. - P. 351-373.

18. Fabry C., Mawhin J. Oscillations of a forced asymmetric oscillator at resonance // Nonlinearity. - 2000. 13. - P. $493-505$.

19. Fabry C., Mawhin J. Properties of solutions of some forced nonlinear oscillators at resonance // Nonlinear Analysis / Eds K. C. Chang and Y. M. Long. - Singapore: World Sci., 2000. - P. 103-118.

20. Fonda A. Positively homogeneous Hamiltonian systems in the plane // J. Different. Equat. - 2004. - 200 - P. $162-184$.

21. Fonda A. Topological degree and generalized asymmetric oscillators // Top. Meth. Nonlinear Anal. - 2006. - 28. - P. $171-188$

22. Fonda A., Mawhin J. Planar differential systems at resonance // Adv. Different. Equat. (to appear).

23. Fučı S. Boundary value problems with jumping nonlinearities // Čas. péstov. mat. - 1976. - 101. P. $69-87$.

24. Fučík S. Solvability of nonlinear equations and boundary value problems. - Boston: Reidel, 1980.

25. Gaines R. E., Mawhin J. Coincidence degree and nonlinear differential equations. - Berlin: Springer, 1977.

26. Kunze M. Remarks on boundedness of semilinear oscillators // Nonlinear Anal. and Appl. Different. Equat. (Lisbon, 1998). - Boston: Birkhäuser, 2001. - P. 311-319.

27. Kunze M., Küpper T., Bin Liu. Boundedness and unboundedness of solutions of reversible oscillators at resonance // Nonlinearity. - 2001. - 14. - P. $1105-1122$.

28. Lazer A. C., Leach D. E. Bounded perturbations of forced harmonic oscillators at resonance // Ann. mat. pura ed appl. - 1969. - 82. - P. 49-68.

29. Xiong $\mathrm{Li}$. Boundedness of solutions for semilinear reversible systems // Proc. Amer. Math. Soc. - 2004. 132. - P. $2057-2066$

30. Xiong $\mathrm{Li}$. Invariant tori for semilinear reversible systems // Nonlinear Anal. - 2004. - 56. - P. 133 - 146.

31. Xiong Li, Qing Ma. Boundedness of solutions for second order differential equations with asymmetric nonlinearity // J. Math. Anal. and Appl. - 2006. - 314. - P. 233 -253.

32. Littlewood J. E. Some problems in real and complex analysis. - Lexington, Mass: Heath, 1969.

33. Bin Liu. Boundedness of solutions for semilinear Duffing equations // J. Different. Equat. - 1998. - 145. - P. $119-144$.

34. Bin Liu. Invariant tori in nonlinear oscillations (Chinese) // Sci. China A. - 1999. - 42. - P. 1047 - 1058.

35. Bin Liu. Boundedness in asymmetric oscillations // J. Math. Anal. and Appl. - 1999. - 231. - P. $355-373$

36. Bin Liu. Boundedness in nonlinear oscillations at resonance // J. Different. Equat. - 1999. - 153. - P. 142 174.

37. Bin Liu. Quasiperiodic solutions of semilinear Liénard equations // Discrete Contin. Dynam. Systems. 2005. - 12. - P. 137 - 160.

38. Lur'e A. I. Some nonlinear problems of the theory of automatic regulation (in Russian). - Moscow; Leningrad: Gostekhizdat, 1951.

39. Massera J. L. The existence of periodic solutions of systems of differential equations // Duke Math. J. 1950. - 17. - P. $457-475$. 
40. Morris G. R. A case of boundedness of Littlewood's problem on oscillatory differential equations // Bull. Austral. Math. Soc. - 1976. - 14. - P. $71-93$.

41. Moser J. On invariant curves of area-preserving mappings of annulus // Nachr. Akad. Wiss. Göttingen. II. Math.-phys. K1. - 1962. - S. 1-20.

42. Ortega R. Asymmetric oscillators and twist mappings // J. London Math. Soc. - 1996. - 53. - P. 325 - 342.

43. Ortega $R$. On Littlewood's problem for the asymmetric oscillator // Rend. Semin. mat. e fis. Milano. 1998. - 68. - P. $153-164$.

44. Ortega $R$. Boundedness in a picewise linear oscillator and a variant of the small twist theorem // Proc. London Math. Soc. - 1999. - 79. - P. 381 - 413.

45. Ortega $R$. Twist mappings, invariant curves and periodic differential equations // Nonlinear Analysis and its Applications to Differential Equations (Lisbon, 1998). - Basel: Birkhäuser, 2001. - P. 85-112.

46. Ortega $R$. Invariant curves of mappings with averaged small twist // Adv. Nonlinear Stud. - 2001. - 1. P. $14-39$.

47. Ortega R. Periodic perturbations of an isochronous center // Qual. Theory Dynam. Systems. - 2002. - 3. - P. $83-91$.

48. Seifert G. Resonance in undamped second-order nonlinear equations with periodic forcing // Quart. Appl. Math. - 1990. - 48. - P. 527 - 530.

49. Dingbian Qian. Resonance phenomena for asymmetric weakly nonlinear oscillator // Sci. China Ser. A. 2002. - 45. - P. 214-222.

50. Zaihong Wang. Multiplicity of periodic solutions of Duffing equations with jumping nonlinearities // Acta Math. Appl. Sin. Engl. Ser. - 2002. - 18. - P. 513-522.

51. Zaihong Wang. Existence and multiplicity of periodic solutions of the second-order differential equations with jumping nonlinearities // Ibid. - P. 615-624.

52. Zaihong Wang. Periodic solutions of the second order differential equations with asymmetric nonlinearities depending on the derivatives // Discrete Contin. Dynam. Systems. - 2003. - 9. - P. 751 - 770.

53. Zaihong Wang. Irrational rotation numbers and unboundedness of solutions of the second order differential equations with asymmetric nonlinearities // Proc. Amer. Math. Soc. - 2003. - 131. - P. $523-531$.

54. Xiaojing Yang. Unboundedness of the large solutions of some asymmetric oscillators at resonance // Math. Nachr. - 2004. - 276. - P. 89 - 102.

55. Xiaojing Yang. Unbounded solutions in asymmetric oscillations // Math. Comput. Modelling. - 2004. 40. - P. $57-62$.

56. Xiaojing Yang. Unbounded solutions of asymmetric oscillator // Math. Proc. Cambridge Phil. Soc. - 2004. - 137. - P. $487-494$.

57. Xiaojing Yang. Existence of periodic solutions in nonlinear asymmetric oscillations // Bull. London Math. Soc. -2005 . - 37. - P. 566-574.

58. Xiaojing Yang. Unbounded solutions of differential equations of second order // Arch. Math. (Basel). 2005. - 85. - P. 460-469.

59. Xiaojing Yang. Existence of periodic solutions of a class of planar systems // Z. anal. Anwend. - 2006. 25. - S. 237-248.

60. Xiaojing Yang. Boundedness in asymmetric oscillators (to appear).

61. Xiaomei Zhang, Dingbian Qian. Existence of periodic solutions for second-order differential equations with asymmetric nonlinearity (Chinese) // Acta math. sinica (Chin. Ser.). - 2003. - 46. - P. 1017 - 1024.

Received 27.10.2006 